\title{
Evaluating the Alarm Fatigue and its Associated Factors among Clinicians in Critical Care Units
}

\author{
Hadia Bourji, Hala Sabbah, Ahmad Al'Jamil, Rania Khamis, Sanaa Sabbah, Nabil Droubi, and \\ Ibtissam Sabbah
}

\section{ABSTRACT}

The Alarm Fatigue (AF) occurs when clinicians are exposed to a large number of false alarms which can cause alarm desensitization. AF is a wellrecognized patient safety concern in intensive care settings. This study aims to evaluate the alarm fatigue in clinicians along with its associated factors in critical care settings using a valid and reliable instrument. During 2020, a cross-sectional study was conducted among clinicians working in Critical care units in Lebanon and enrolled 337 participants via self-administered electronic questionnaire. The survey included questions on sociodemographic characteristics, health status, staff recognition of alarms and alarm management, and Alarm Fatigue questionnaire. The translation and cultural adaptation of the Alarm Fatigue questionnaire in Arabic followed a standardized protocol. The mean age of participants was 30.79 ( $\mathrm{SD}=7.26$; median=29) years, $61.4 \%$ were women. $77.2 \%$ were nursing staff and $22.8 \%$ were physicians. $\mathbf{7 8 . 8 \%}$ worked in private hospitals. $41.3 \%$ of staff were from neonatal and pediatric intensive care units. $58.8 \%$ of clinicians find that more than $50 \%$ of alarms were irrelevant, and $69.4 \%$ deactivate and silence the alarm when annoyed. The Alarm Fatigue questionnaire has good reliability and validity. The Cronbach alpha was 0.69 which establishes good reliability for group comparison. $A F$ in physicians $(\mathrm{OR}=2.25$; 95\% $\mathrm{CI}=-\mathbf{0 . 0 1 - 4 . 5 0}, \mathrm{p}=\mathbf{0 . 0 5})$ and Registered Nurses $(\mathrm{OR}=2.64 ; 95 \% \mathrm{CI}=-$ $0.52-4.75, p=0.02$ ) was higher than in nurses and head nurses; $\mathrm{AF}$ was more frequent in ICUs units $(\mathrm{OR}=\mathbf{2 . 1} ; \mathbf{9 5 \%} \mathrm{CI}=\mathbf{0 . 0 5}-4.18, \mathrm{p}=\mathbf{0 . 0 4})$ compared to other critical care units. The AF was higher for the clinicians that deactivate and silence the alarm when annoyed compared to other subgroup $(\mathrm{OR}=4.14,95 \% \mathrm{CI}=2.75-5.53, \mathrm{p}<0.001)$. The $\mathrm{AF}$ was 3.14 times frequent for the staff who reported stress $(\mathbf{p}<0.001)$. Non-actionable alarms occurred frequently and associated to alarm fatigue. Physicians score slightly higher alarm fatigue than nurses. The Arabic Alarm Fatigue questionnaire has a good psychometric properties and suitable to evaluate the AF in Lebanon. Multidisciplinary approaches can be established to minimize the incidence of $\mathrm{AF}$ using a valid and reliable instrument.

Keywords: Alarm Fatigue questionnaire, clinicians, clinical alarms, critical care units.

\section{INTRODUCTION}

Alarm fatigue is a well-recognized patient safety concern in intensive care settings [1]. Critical care unit can be defined as a place where patients with advanced medical conditions can be monitored in a well-established system and by advanced machines [2]. The use of sophisticated machines designated to alarm for serious changes in patients' statuses in these units is common. These devices communicate the changes in status of the patients to the staff by alarm signals descript by clinical alarms [2]- [5]. However, technical alarms indicate that the biomedical equipment requires attention [4], [5].
Published Online: December 27, 2020

ISSN: $2736-5476$

DOI: $10.24018 /$ clinicmed.2020.1.1.8

Hadia Bourji

Master student, Faculty of Public Health, Lebanese University, Saida, Lebanon. (e-mail: hadiabourgi@outlook.com) Hala Sabbah

Faculty of Economic Sciences and Business Administration, Lebanese University, Nabatieh, Lebanon.

(e-mail: hsabbah@ul.edu.lb)

Ahmad Al'Jamil

Master student, Faculty of Public Health, Lebanese University, Saida, Lebanon. (e-mail: Ahmed.Aljamil@st.ul.edu.lb) Rania Khamis

Institute of Social Science, Lebanese University, Saida, Lebanon.

(e-mail: Rania.khamis@ul.edu.lb ) Sanaa Sabbah

Institute of Social Science, Lebanese University, Saida, Lebanon.

Doctoral School of Literature, Humanities \& Social Sciences, Lebanese University, Beirut, Lebanon.

(e-mail: Sanaa.Sabbah_@ul.edu.lb )

Nabil Droubi

MD, Saida, Lebanon.

(e-mail: nsdroubi_@inco.com.lb)

Ibtissam Sabbah*

Faculty of Public Health, Lebanese University, Saida, Lebanon.

(e-mail: ibtissam.sabbah@ul.edu.lb)

*Corresponding Author

Clinical alarm systems safety has received immense attention from clinicians, patient safety organizations, and policy makers [6]. In hospital intensive care units, the monitor devices aid clinicians hugely in taking instantaneous actions [7]. Indeed, critical care unit is a place where clinical alarms occur frequently [2].The alarms of equipment intended to protect patients increase noise within the unit. The average sound levels always exceeded 45 decibels A (dBA) and for $50 \%$ of the time exceeded between 52 and $59 \mathrm{dBA}$ in individual ICUs [8], [9]. These sound pressure levels clearly exceed those of $35 \mathrm{dBA}$ recommended by the World Health Organization (WHO) for nighttime in hospitals [9]. 
Noise affects patients and staff. It disrupts rest, impedes concentration and cognition [10], [11], disrupts sleep [12], interferes with communication [11] and increases the risk of accidents. The excessive number of such alarms with low clinical relevance [3], [10] leads to what is called 'alarm fatigue'. Alarm Fatigue occurs when healthcare providers are exposed to a large number of false alarms which can cause alarm desensitization [2], [10], [13]. That is, the clinician familiarizes within the working environment with the alarm sounds as background noise [7]. The frequent false alarms may produce the "cry wolf" effect. Healthcare providers (nurses or physicians) grow accustomed to their voices and might regard significant alarms as false, thus, failing to respond properly [4], [14], [15]. It may also make an alarm system less reliable [14]. The researchers demonstrated that $72 \%-99 \%$ of the alarms are false [3], [5].

Alarm overload and "Alarm fatigue" (AF) are conditions that may lead to incidents [5], [10], [16]. When fatigued by alarms, the staff may deactivate variables that need to be monitored, lower the volume, silence, disable alarms or inadvertently adjust their parameters beyond the limits appropriate for the patients' needs in an attempt to decrease the number of alarms [10], [13]. In fact, clinicians if extremely disturbed from alarms, could ignore and silence them at the central station [7]. Such changes may contribute to missing serious and important changes in a patient's condition [10], [13]. Alarm fatigue is a kind of human error [3], [16]. Ultimately, consequences of AF include missed alerts of clinical significance, with substantial risk for patient harm and potentially fatal outcomes [15], [17]. Alarm fatigue may also cause a 'second victim' effect. One of the authors knows from personal experience two hospitals in which a staff member was rendered unfit for work for several months after a fatal accident caused by alarm fatigue [18].

Recent studies in Critical care units show that alarm safety is affected by multifactor such as lack of policies and procedures on alarm management, poor usability of physiologic monitors, lack of standardized practices on alarm reduction strategies, and lack of nurse competence on physiologic monitors use [10]. Factors related to the environment and organizations where the alarms were located such as bed to alarm ratio, purpose and length of alarm conditions, staffing levels, high-risk patient ratio required intense monitoring [5]. The unit layout and background noise were also considered important [5], [10], [19]. The improvement of the safe use of healthcare technology requires that diverse stakeholders come together in order to utilize their collective knowledge on the design, use, integration, and servicing of healthcare technology, systems, and devices [15]. Measurement of staff response to alarm signals was suggested to help to understand inconsistent staff behavior in responding to alarm signals [5]. Though a number of interventions have been suggested to reduce the number of false alarms, there is a need for more thorough research to address the possible negative consequences of such interventions. Based on the results of several studies in the fields of alarm fatigue and alarm management, a variety of measures have been suggested to lessen alarm fatigue [16]. So, there is need for valid, reliable, and transferable instruments to measure alarm fatigue in health professionals [14], [16]. In Lebanon, there are a scarcity of research conducted on medical device alarms, and alarm fatigue is a newly introduced concept. There are not any comprehensive instruments exclusively designed for measuring alarm fatigue in healthcare settings. The research question is: are clinicians (nurses and physicians) working in critical care units at risk for and experiencing alarm fatigue? Which devices are the most concerned in alarm fatigue? Do irrelevant alarms and nuisances contribute to morbidities among clinicians?

This study aims to evaluate the alarm fatigue in healthcare providers (nurses or physicians) along with its associated factors in critical care settings using a valid and reliable instrument. This information will provide basic data that could help create a safe hospital environment.

\section{MATERIAL AND METHODS}

\section{A. Study Design}

From June until August 2020, a cross-sectional study was conducted among healthcare providers working in Critical care units in Lebanon and enrolled 337 participants. 16 hospitals were selected to represent all Lebanese hospitals using a simple random sampling method. All consenting nurses and physicians working in critical care units at the selected hospital facilities were eligible to participate in the study. The inclusion criteria consist of all nurses employed as registered nurses and physicians working on the study units at the time of the observations and agreed to participate in the study. The exclusion criteria included nursing and medical students and practical nurses.

The approval of the ethical committee of Lebanese University was obtained for the research plan (No.) before the research began. Written informed consent was obtained from all participants. A cover letter accompanied the survey explaining that participation was voluntary and that the survey was anonymous. In addition, managers of hospitals and /or study units provided a written agreement of participation. Due to constraints from Covid-19 pandemic, data were collected via online questionnaire (Google Form). The link to the questionnaire was distributed by the researchers via social media platforms. A pilot study on 10 individuals was conducted beforehand to test the feasibility of the questionnaire.

The sampling method was non-probability, convenience sampling. The sample size of nurses was calculated according to Bartlett, Kotrlik, and Higgins table [20] using Cochran's formulas, selecting an alpha level of 5\%, a margin of error of .05 , and a population size approximately of 10000 subjects (at the end 2019, the total number of nurses working in hospital sector was 9800 [21]; the rate of nurses working in critical care unit is unknown). This led us to predict that 264 subjects would be necessary for the survey. Concerning physicians, a sample of 77 was selected in a nonrandom manner (purposive sampling) in order to obtain a sample of medical staff that represents the physicians working in critical care units.

\section{B. Questionnaire}

All data was collected utilizing a questionnaire containing the following parts:

The first part of the questionnaire, developed by the researchers, consisted of sociodemographic status, including 
age, gender, marital status, educational level, and job position. Data were also collected regarding type of hospital, work unit, years of overall experience in work, years of overall ICU experience, and the ratio of nurses per patient.

Section 2 included questions about monitoring equipment and alarm management. We assessed data about the rate of non-actionable alarms (false, technical or nuisance alarms) [17]. Also, data of types of medical devices and alarms that mostly emit irrelevant alarms causing highest alarm fatigue were collected. The alarm management by the medical and nurse's staff was also assessed by asking if they deactivate or put the alarms on silent when disturbed. The clinician responsible to adjust the parameters of the clinical alarms, if alarms reduce trust in alarm systems, and whether there exist policies and procedures on alarm management in the studied facilities were also collected. Data also assessed the impact of alarms on health (genesis of stress or anxiety, sleep disturbance, headache) and the quality of life of the participants.

The alarm fatigue was evaluated using the Alarm fatigue questionnaire that was developed by Torabizadeh and colleagues [16]. Permission was taken from the authors to use the instrument and to apply some adaptations. The questionnaire consisted of 13 questions scored on a five-point Likert scales: never, rarely, occasionally, usually, and always. Each item on the questionnaire is scored from 0 ("never") to 4 ("always"), (except items 2 and 9 which are scored reversely). The scores of items are summed to give an overall score. The score range of the questionnaire is between 8 (minimum) and 44 (maximum) with higher scores indicating higher levels of Alarm fatigue and indicating a greater impact of alarm fatigue on participants' performance. It is a validated and reliable questionnaire. The content validity index of the questionnaire was 0.92 [16]. The Alpha Cronbach coefficient for the overall scale of the Alarm fatigue questionnaire was 0.91 , which is an indicator of the reliability of the scale [16].

In this study, the translation and cultural adaptation of the Alarm fatigue questionnaire in Arabic followed a standardized protocol [22]. No-one refused to answer the questions of the Alarm fatigue questionnaire. There were no missing data or incomprehensible items, and the duration of administration of the questionnaire was short (10 minutes). This reinforces the face validity and therefore makes it possible to approve the absence of problems related to translation [22]. The Cronbach alpha was 0.69 which establishes good reliability (an alpha of greater than .50) for group comparison [23]. The presence of a relation between the scores of Alarm fatigue questionnaire and the characteristics of the respondents supports the construct validity of the instrument [22], [23]. We hypothesized that the Alarm fatigue total scores differ among healthcare providers subgroups and that these would be associated with characteristics, health status, and quality of life perception of the respondents, and the recognition of alarms and alarm management by the medical and nurse's staff. Indeed, the results of Principal Component Analysis (PCA) suggested possible existence of two underlying factors according to the factor loadings of the 13 items (results not shown in this manuscript) that it was in line with the original scale findings [16]. The Arabic Alarm fatigue questionnaire has been evaluated as a valid and reliable instrument. Thus, it may be suitable for evaluating the Alarm fatigue of Lebanese healthcare providers in critical care settings

\section{Data Analysis}

The sample was described using mean, medians with interquartile ranges (IQRs) for scores of the overall Alarm Fatigue questionnaire and quantitative variables, given that they were mostly not normally distributed, and using numbers and percentages for qualitative variables and the scores of items of alarm fatigue questionnaire classified by level. The normality of the distribution was checked using the ShapiroWilk test (SW) test [24]. The Significance value of the SW test $(\mathrm{p}<0.001)$ is below 0.05 , so the data significantly deviate from a normal distribution. The Differences of alarm fatigue scores between groups was assessed using Mann-Whitney and Kruskal-Wallis tests when appropriate. A p-value less than 0.05 is to be considered significant.

Multivariate analysis of variance was performed (General Linear Model) to determine the potential risk factors of reporting alarm fatigue. All explanatory variables with significance level is less than or equal 0.05 in bivariate analysis were included except education level because of its association with the job position. Associations between explanatory variables and alarm fatigue scores are presented as odds ratios (ORs) and 95\% confidence intervals (CIs).

Psychometric properties of the survey of internal consistency, reliability, and construct validity were assessed as part of the study. The collected data were analyzed using SPSS ver. 21.0 for Windows (SPSS Inc., Chicago, IL, USA).

\section{RESULTS}

The study was conducted in 16 critical care facilities in Lebanon. A total of 337 subjects agreed to participate in this study. No questionnaire was excluded from the analysis because of missing data. $77.2 \%$ of participants were nursing staff and $22.8 \%$ were physicians. The majority (78.8\%) works in private hospitals. The mean age was $30.79(\mathrm{SD}=7.26$; median $=29$ ) years, with approximately $78.5 \%$ being younger than 40 years. $61.4 \%$ were women and $43.9 \%$ were married. Nearly all of the respondents $(94.4 \%)$ had a university level degree and higher. The mean years of professional experience was 8.47 ( $\mathrm{SD}=6.58$; median=7). The minimum level of the respondent's experience was one year, and the maximum was 40 years. The majority work full-time $(91.4 \%)$. However, one third $(40.1 \%)$ of staff were within $1-3$ years of critical care experience. The staff worked in various critical care units. $41.3 \%$ of staff were from neonatal and pediatric intensive care units. Two third (63.5\%) worked as Registered Nurses (RN). $22.8 \%$ of the respondents worked less than 40 hours per week, $58.7 \%$ worked between 40 and 48 hours while the remainder (18.4\%) worked 48 hours and higher weekly.50.1\% worked a supplementary hours ranged between 7-36 hours weekly. $94.2 \%$ of staff take in charge 3 patients (patient ratio of 1:3.) and more per shift. Headache $(84.0 \%)$ and insomnia $(78.9 \%)$ were the predominant declared symptoms, whereas stress was reported by $74.8 \%$ of the study participants (Table 1). 
TABLE 1 DETAILED CHARACTERISTICS OF THE STUDY SAMPLE (N=337) Frequency (\%)

\begin{tabular}{|c|c|}
\hline \multicolumn{2}{|l|}{ Age group (years) } \\
\hline $20-29$ & $176(52.2)$ \\
\hline 30- 39 & $119(35.3)$ \\
\hline $40-49$ & $33(9.8)$ \\
\hline $50-60$ & $9(2.7)$ \\
\hline Gender (female) & $207(61.4)$ \\
\hline \multicolumn{2}{|l|}{ Marital status } \\
\hline Single & $164(48.7)$ \\
\hline Married & $148(43.9)$ \\
\hline Divorced or widowed & $25(7.4)$ \\
\hline \multicolumn{2}{|l|}{ Education } \\
\hline Baccalaureate & $19(5.6)$ \\
\hline Bachelor degree & $196(58.2)$ \\
\hline Master degree & $45(13.4)$ \\
\hline $\mathrm{PhD}$ in medicine & $27(8.0)$ \\
\hline Specialist physician & $50(14.8)$ \\
\hline \multicolumn{2}{|l|}{ Job position } \\
\hline Nurse & $19(5.6)$ \\
\hline Registered nurse & $214(63.5)$ \\
\hline Head Nurse, supervisor & $27(8.0)$ \\
\hline Medical staff & $77(22.8)$ \\
\hline Type of hospital & $233(69.1)$ \\
\hline Teaching Hospital & $104(30.9)$ \\
\hline \multicolumn{2}{|l|}{ General purpose Hospital } \\
\hline \multicolumn{2}{|l|}{ Critical care unit } \\
\hline ICU / CCU & $72(21.4)$ \\
\hline PICU / NICU & $28(8.3)$ \\
\hline $\mathrm{CCU}$ & $41(12.2)$ \\
\hline ICU & $85(25.2)$ \\
\hline NICU & $76(22.6)$ \\
\hline PICU & $35(10.4)$ \\
\hline \multicolumn{2}{|l|}{ Years of overall ICU experience } \\
\hline$<1$ year & $45(13.4)$ \\
\hline $1-3$ years & $90(26.7)$ \\
\hline 4-9 years & $133(39.5)$ \\
\hline 10 years and highest & $69(20.5)$ \\
\hline \multicolumn{2}{|l|}{ Shift duty } \\
\hline Day & $98(29.1)$ \\
\hline Night & $45(13.4)$ \\
\hline Alternant & $194(57.6)$ \\
\hline \multicolumn{2}{|l|}{ Ratio nurses per patient } \\
\hline $1-2$ & $23(6.8)$ \\
\hline 3 & $147(43.6)$ \\
\hline Above 3 & $167(49.6)$ \\
\hline Sleep disturbance (yes) & $266(78.9)$ \\
\hline Headache (yes) & $283(84.0)$ \\
\hline Anxiety and stress (yes) & $252(74.8)$ \\
\hline Quality of life perception (bad) & $275(81.6)$ \\
\hline
\end{tabular}

The participants declared the types of alarms and monitoring devices with the highest non actionable alarm frequencies and greatest impact on $\mathrm{AF}$ : mechanical ventilators $(72.1 \%)$, saturation monitoring (SPO2) (77.2\%), invasive blood pressure monitoring (61.7\%), electrocardiographic and blood pressure monitoring (72.1\%). The noise of phones ringing in the critical care settings was declared by $60.5 \%$ of clinicians (Figure 1). Each of these factors contributes to alarm fatigue $(\mathrm{p}<0.001) .69 .4 \%$ of clinicians deactivate and silence the alarms when they annoy them. $72.7 \%$ declared that nurses are allowed to adjust the alarm parameters to suit the patient's clinical situation. Only $50.1 \%$ declared that alarm management policies and procedures were available in their units (Table 2).

Clinicians scored highest for "I believe much of the noise in the ward is from the alarms of the monitoring equipment" and lowest for "I react differently to low volume (yellow) and high volume (red) alarms from medical devices" (Figure 2).

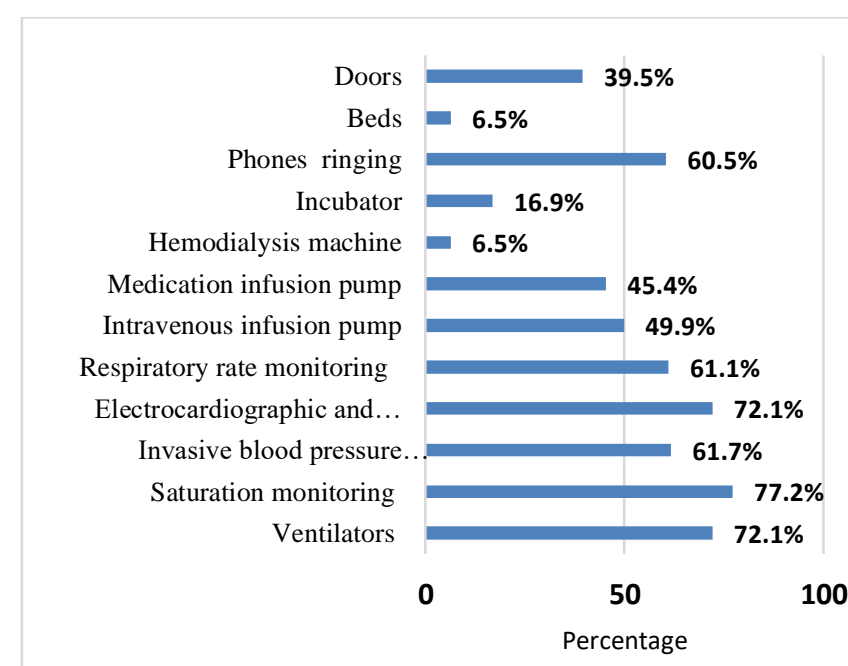

Fig.1. Devices that mostly emit irrelevant alarms and most concerned in Alarm fatigue

Clinicians scored highest for "I believe much of the noise in the ward is from the alarms of the monitoring equipment" and lowest for "I react differently to low volume (yellow) and high volume (red) alarms from medical devices" (Figure 2). The median of all items was 3 except the item 9 (I react differently to low volume (yellow) and high volume (red) alarms from medical devices) where the median was 1 ; in addition, the median was 2 for the following items: 1(I regularly readjust the limits of alarms based on the clinical symptoms of patients), 10 (when I'm upset and nervous, I'm more responsive to alarm sounds), and 13 (at visiting hours, I pay less attention to the alarms of the equipment) they medians was 2). Table 3 presents the descriptive statistics of the Alarm fatigue questionnaire of the respondents. The mean of the overall alarm fatigue was 30.57 ( $\mathrm{SD}=7.89$; median=31; minimum=12; maximum=44.00). The Mean Alarm Fatigue score was higher in males as compared to females $(\mathrm{p}=0.002)$. In general, in this study physicians reported higher level of alarm fatigue than nurses $(\mathrm{p}=0.02)$. Alarm fatigue was highest among staff working in general purpose hospitals $(\mathrm{p}=0.02)$ compared to teaching hospitals; however, no differences were observed between private and public sector. Alarm fatigue proved to be higher in night shift compared to rotating and daytime workers $(\mathrm{p}=0.03)$. Statistically significant relationships were found between health status (declared symptoms), quality of life perception, and alarm fatigue $(\mathrm{p}<0.001)$ (Table 3). Staff who reported that they are bothered by the alarms and put alarms in silent condition in their shift reported high level of alarm fatigue $(p<0.001)$. The scores of alarm fatigue increased according to the rate of perceived non actionable alarms by the healthcare providers: the clinicians who perceived that over $70 \%$ of alarms as irrelevant reported more alarm fatigue than others $(\mathrm{p}<0.001)$ subgroups (Table 2 ). There was no association between healthcare providers' alarm fatigue and age groups $(\mathrm{p}=0.69)$, years of experience $(\mathrm{p}=0.40)$, and family situation $(\mathrm{p}=0.60)$, and the ratio per patient $(\mathrm{p}=0.12)$. These results support the construct validity of the alarm fatigue scale. 
TABLE 2 ALARM FATIGUE ACCORDING TO CHARACTERISTICS AND MANAGEMENT OF ALARMS Alarm fatigue scale

\begin{tabular}{lccc}
\hline & Frequency (\%) & Mean (SD) & $\begin{array}{c}\text { Median (interquartile } \\
\text { ranges) }\end{array}$ \\
\hline Rate of non-actionable alarms & & & $<0.001$ \\
Less than 30\% of alarms & $65(19.3)$ & $24.00(5.81)$ & $24(8.5)$ \\
Between 30\% and 50\% of alarms & $74(22.0)$ & $25.07(5.68)$ & $24(8.0)$ \\
Between 50\% and 70\% of alarms & $72(21.4)$ & $33.14(6.71)$ & $36(11)$ \\
Over 70\% of alarms & $126(37.4)$ & $35.73(5.87)$ & $37(5)$ \\
\hline Deactivation and silencing the alarm & & & $36(11)$ \\
Yes & $234(69.4)$ & $33.43(6.77)$ & $23(8)$ \\
No & $103(30.6)$ & $24.09(6.24)$ & $<0.001$ \\
\hline Responsible of alarm limit settings & & & \\
Nurse & $245(72.7)$ & $29.92(7.83)$ & $31(14)$ \\
physician & $92(27.3)$ & $32.30(7.81)$ & $34.5(12.8)$ \\
\hline policies and procedures on alarm management & & & \\
Yes & $169(50.1)$ & $27.36(7.72)$ & $26(13)$ \\
No & $168(49.9)$ & $33.81(6.65)$ & $36(9.8)$ \\
\hline Alarms reduce trust in alarm systems & & & \\
Yes & $253(75.1)$ & $32.05(7.40)$ & $35(12)$ \\
No & $84(24.9)$ & $26.12(7.66)$ & $25(9.8)$ \\
\hline
\end{tabular}

TABLE 3. ALARM FATIGUE ACCORDING TO THE RESPONDENTS CHARACTERISTICS' (N=337)

\begin{tabular}{|c|c|c|c|}
\hline & \multicolumn{3}{|c|}{ Alarm fatigue scale } \\
\hline & Mean (SD) & Median (IQR) & $P$ value \\
\hline Gender & & & 0.002 \\
\hline Male & $32.26(7.72)$ & $35(11.25)$ & \\
\hline Female & $29.51(7.82)$ & $29(14)$ & \\
\hline Education & & & 0.03 \\
\hline Baccalaureate & $31.26(7.29)$ & $36(13)$ & \\
\hline Bachelor degree & $29.50(8.04)$ & $29(14)$ & \\
\hline Master degree & $31.07(7.38)$ & $32(12.5)$ & \\
\hline $\mathrm{PhD}$ in medicine & $32.56(7.18)$ & $34(12)$ & \\
\hline Specialist physician & $33.00(7.69)$ & $35.5(12)$ & \\
\hline Job position & & & 0.02 \\
\hline Nurse & $31.26(7.47)$ & $36(13)$ & \\
\hline Registered nurse & $29.90(7.88)$ & $30(14)$ & \\
\hline Head Nurse, supervisor & $28.96(8.44)$ & $29(16)$ & \\
\hline medical staff & $32.84(7.47)$ & $35(12)$ & \\
\hline Type of hospital & & & 0.02 \\
\hline Teaching private & $30.56(7.91)$ & $31(14)$ & \\
\hline Teaching governmental & $27.44(8.48)$ & $25(16)$ & \\
\hline General $^{1}$ private & $31.22(7.29)$ & $33(11.5)$ & \\
\hline General governmental & $33.45(7.14)$ & $36(10)$ & \\
\hline Critical care unit & & & 0.04 \\
\hline $\mathrm{ICU} / \mathrm{CCU}$ & $28.72(8.42)$ & $28(14)$ & \\
\hline PICU / NICU & $32.46(7.94)$ & $35.5(11.75)$ & \\
\hline $\mathrm{CCU}$ & $31.80(6.89)$ & $34(10.5)$ & \\
\hline $\mathrm{ICU}$ & $31.67(7.29)$ & $32(13)$ & \\
\hline NICU & $28.92(7.90)$ & $27(13)$ & \\
\hline PICU & $32.34(8.19)$ & $35(16)$ & \\
\hline Years of ICU experience & & & 0.001 \\
\hline$<1$ year & $32.78(7.57)$ & $35(14.5)$ & \\
\hline $1-3$ years & $28.76(7.96)$ & $27(13)$ & \\
\hline $4-9$ years & $32.01(7.36)$ & $34(12)$ & \\
\hline 10 years and highest & $28.74(8.19)$ & $28(14.5)$ & \\
\hline Shift duty & & & 0.03 \\
\hline Day & $30.90(8.02)$ & $33(13)$ & \\
\hline Night & $33.00(7.17)$ & $35(11.5)$ & \\
\hline Alternant & $29.85(7.89)$ & $29.5(14)$ & \\
\hline Sleep disturbance & $32.08(7.45)$ & $34(12)$ & $<0.001$ \\
\hline Yes & $24.93(6.90)$ & $24(9)$ & \\
\hline No & & & \\
\hline Headache & $31.70(7.64)$ & $34(13)$ & $<0.001$ \\
\hline Yes & $24.65(6.39)$ & $23.5(7.25)$ & \\
\hline No & & & \\
\hline Anxiety and stress & $32.76(7.01)$ & $34(12)$ & $<0.001$ \\
\hline Yes & $24.08(6.69)$ & $24(9.0)$ & \\
\hline No & & & \\
\hline Quality of life perception & & & $<0.001$ \\
\hline $\mathrm{Bad}$ & $32.05(7.44)$ & $34(13)$ & \\
\hline No bad & $24.00(6.34)$ & $23(9)$ & \\
\hline Total sample & $30.57(7.89)$ & $31(14)$ & \\
\hline
\end{tabular}

Notes and abbreviations: 1 general-purpose= general; Intensive care unit (ICU); Coronary care unit (CCU); ICU / CCU combined; Pediatric Intensive Care Unit (PICU); Neonatal Intensive Care Unit (NICU); PICU /NICU combined. Interquartile ranges (IQRs). 
Multivariate analysis showed that the level of alarm fatigue is not significantly different between males and females $(\mathrm{p}=0.09)$. Alarm fatigue in physicians $(\mathrm{OR}=2.25$; $95 \% \mathrm{CI}=-$ $0.01-4.50, \mathrm{p}=0.05)$ and $\mathrm{RNs}(\mathrm{OR}=2.64 ; 95 \% \mathrm{CI}=-0.52-4.75$, $\mathrm{p}=0.02$ ) was higher than in nurses, head Nurses, and supervisors. Alarm fatigue was more frequent in ICUs units $(\mathrm{OR}=2.1 ; 95 \% \mathrm{CI}=0.05-4.18, \mathrm{p}=0.04)$ compared to other critical care units.

Alarm fatigue was more frequent for subjects working in critical care units where the physician is the only responsible of alarm limit settings $(\mathrm{p}=0.01)$ and far less frequent in subjects working in units that have policies and procedures on alarm management $(\mathrm{p}=0.004)$ and who reported a rate of non-actionable alarms lower than $50 \%$ of alarms $(\mathrm{p}<0.001)$.

The alarm fatigue was higher for the clinicians that deactivate and silence the alarm when annoyed compared to other subgroup or those who were not deactivate the alarms $(\mathrm{OR}=4.14,95 \% \mathrm{CI}=2.75-5.53, \mathrm{p}<0.001)$. The alarm fatigue was associated with stress: alarm fatigue was 3.14 times frequent for the staff who reported stress $(p<0.001)$ and was more frequent in subjects who reported an impact of the alarm on their quality of life than those who reported any impact of the alarms on their quality of life $(\mathrm{OR}=2.86,95 \% \mathrm{CI}=1.08$ 4.64, p<0.001) (Table 4 )

\section{DISCUSSION}

To our knowledge, this is the first Lebanese study evaluating the $\mathrm{AF}$ and reported practices toward alarms management. Among the identified explanatory factors, being physician or registered nurse, having self-reported stress, anxiety, and a low quality of life were associated with high level of alarm fatigue. In this study, it was shown that the AF is more problematic for clinicians working in ICUs Units, having lack of alarm policies in their facilities, and those that deactivate and silence the alarm when annoyed.

In similarity with our findings previous study showed that nurses felt alarm fatigue [14]. In addition, the most of nurses did not recognize how to avoid alarm fatigue [25]. AF can be associated with serious safety risks for clinicians and patients. Based on the survey, $74.8 \%$ of nurses and medical staff report that they are stressed by the alarms and $78.9 \%$ had sleep disturbance. The AF also affects the clinicians' quality of life. This finding agreed with what they reported that alarm fatigue negatively influences the cognitive performance and psychological health of healthcare professionals [11], [18]. The fatigue is a domain of mental health that is very crucial to health related quality of life of the healthcare providers [23]. In the long-term, fatigue can also affect staff health and morale, with effects on cardiovascular outcomes, depression [26], leading to stress [1], [17], and even "burn-out" [17], [26], [27]. Furthermore, stress certainly influences AF by forcing healthcare providers to instantaneously adjust their work activities (and priorities) according to perceived importance of near constant clinical alarm activity [17].

Our findings showed that night shifts are related to alarm fatigue, and may also increase the risk of stress and less time sleeping [27]. In addition, noise has a consequence on staff health and well-being [28]. Indeed, in addition to the circadian alterations described in critical care units' nurses and physicians as well [28], several consequences included the overall shift work sleep disorder class (i.e. reduced work performance, processing errors, accidents, reduced quality of life) are direct or indirect consequences of passing the night in a hostile habitat, even while working in it [28]. So, shifts should always be organized, full weeks of nights are not recommended, and an ideal pattern would see staff work no more than three or four night shifts at a time [26]. The Health and Safety Executive in the United Kingdom, when discussing fatigue management, suggests that if shifts are a maximum of 8 hours, they require special attention and high concentration [26]. Reducing the noise at night results in better sleep for staff and patients [17].

In the present study, approximately half $(58.8 \%)$ of respondents find that more than $50 \%$ of alarms were irrelevant. Our results were lower than reported by previous research [14] that reported that the rate of false alarms was 63.8\%; the American Association of Critical -Care Nurses [29] found that from $89 \%$ to $99 \%$ of electrocardiographic (ECG) monitor alarms were found false or clinically insignificant. Our finding was consistent with that concluded that non-actionable alarms occurred frequently [14], [18], [29]- [31] increase the level of clinicians' alarm fatigue [14], [19], [25] disrupt patient care [18], [25], [31] and reduce trust in alarm systems [19]. Johnson et al, [31] found that the high burden of NICU alarms affects the ability of nurses to both respond to alarms and perform their primary tasks in patient care, assessments, medication administration, infant feeding, documentation [31]. Özcan and Gommers [32] showed that if nurses let the non-actionable alarms sound for an extended period of time, tension amongst nurses arises. Also, Keith and Hueske-Kraus [18] reported that the sounds of alarms reduce the amount of time spent by the physicians on clinical tasks after an interruption [18].

This finding was consistent with results of the American Association of Critical -Care Nurses [29] that concluded to reduce alarms and alarm fatigue, clinical strategies have to focus on ECG and oxygen saturation $\left(\mathrm{SpO}_{2}\right)$ alarms [29]. In Korea, false alarms of the patient monitors accounted for $62.5 \%$ of all the alarms [14]. Thus, it can be inferred that there is no significant difference in the types of alarmgenerating medical devices in critical care units in Lebanon and abroad [14], [29]. Our findings are in line with those of Nguyen et al., [17] that reported the commonly seen reactions to AF including the deactivation and silencing of systems or adjustment of alarm parameters by the healthcare providers to decrease the number of alarms. Further studies showed that the frequent false alarms cause reduced attention or incremental increases in the response time to an alarm [11] [18] or no response at all [1]. Previous study showed that alarms in the critical care unit are most bothersome when nurses need to focus on cognitively demanding tasks [32] such as conversations with visitors, doctors and other nurses, doing administration or having to prepare medication [32]. Other research affirms that improving alarm reliability increases alarm response [30]. Furthermore, in harmony with our results, the decreased clinicians responsiveness and slow response times to alarms are the possible dangerous consequences of alarm fatigue [1], [17]. 
Description of the items in $\%$

At visiting hours, I pay less attention to the alarms of the equipment

Alarm sounds prevent me from focusing on my professional duties

When alarms go off repeatedly and continuously, I lose my patience

When I'm upset and nervous, I'm more responsive to alarm sounds

I react differently to low volume (yellow) and high volume (red)

Alarm sounds make me nervous

When alarms go off repeatedly, I become indifferent to them

In some shifts the heavy workload in the ward prevents my quick.

I pay more attention to the alarms in certain shifts

I believe much of the noise in the ward is from the alarms of the

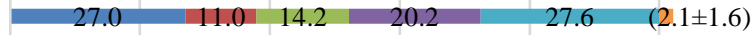

\begin{tabular}{|l|l|l|l|l}
10.7 & 11.6 & 23.1 & 18.7 & 35.9
\end{tabular}

$\begin{array}{llllll}11.3 & 16.0 & 17.5 & 19.0 & 36.2 & (2.5 \pm 1.4)\end{array}$

\begin{tabular}{|l|l|l|l|l|l|}
\hline 21.1 & 18.1 & 17.8 & 23.7 & 19.3 & $(2.0 \pm 1.4)$
\end{tabular}
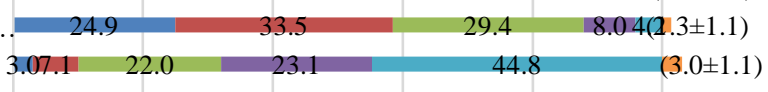

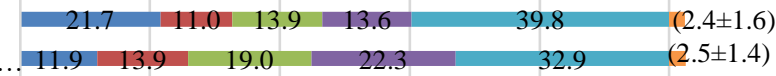

\begin{tabular}{|l|l|l|l|l|}
\hline 6.2 & 15.1 & 14.5 & 27.9 & 36.2
\end{tabular}

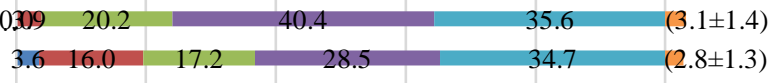

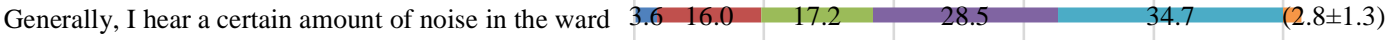

\begin{tabular}{|l|l|l|l|l|l|}
\hline I turn off the alarms at the beginning of every shift & 44.8 & 4.76 .5 & 21.7 & 22.3 & $(1.7 \pm 0.8)$
\end{tabular}

I regularly readjust the limits of alarms based on the clinical symptoms.

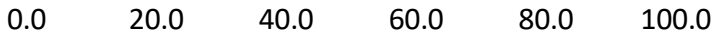

$\square$ Never $\square$ Rarely $\square$ Sometimes $\square$ Often $\square$ Always mean \pm SD ()

Fig. 2. Description of the items of the Alarm Fatigue scale

TABLE 2 THE FACTORS ASSOCIATED WITH ALARM FATIGUE SCORES OF THE CLINICIANS' SUBGROUPS USING MULTIVARIATE ANALYSIS (GLM)

\begin{tabular}{|c|c|c|c|c|}
\hline \multirow[t]{2}{*}{ Parameter } & \multirow[t]{2}{*}{$\mathrm{B}$} & \multirow[t]{2}{*}{$\mathrm{P}$ value } & \multicolumn{2}{|c|}{ 95\% Confidence Interval } \\
\hline & & & Lower Bound & Upper Bound \\
\hline Intercept & 24.09 & $<0.001$ & 20.58 & 27.60 \\
\hline Gender & & 0.09 & -2.28 & 0.17 \\
\hline Female & -1.06 & & & \\
\hline Male & 1 & . & . & . \\
\hline \multicolumn{5}{|l|}{ Job position } \\
\hline Physician & 2.25 & 0.05 & -0.01 & 4.50 \\
\hline Nurse & 2.71 & 0.08 & -0.36 & 5.77 \\
\hline Registered Nurse & 2.64 & 0.02 & 0.52 & 4.75 \\
\hline Head Nurse, supervisor & 1 & NA. & . & . \\
\hline Type of hospital & & 0.31 & & \\
\hline Teaching Hospital & -0.62 & & -1.81 & 0.57 \\
\hline General purpose Hospital & 1 & . & . & . \\
\hline $\begin{array}{l}\text { Critical care unit } \\
\text { ICU / CCU }\end{array}$ & -0.78 & 0.47 & -2.88 & 1.32 \\
\hline PICU / NICU & 1.85 & 0.15 & -0.67 & 4.37 \\
\hline $\mathrm{CCU}$ & 1.27 & 0.28 & -1.06 & 3.60 \\
\hline $\mathrm{ICU}$ & 2.11 & 0.04 & 0.04 & 4.18 \\
\hline NICU & 0.42 & 0.69 & -1.64 & 2.48 \\
\hline PICU & 1 & . & . & . \\
\hline \multicolumn{5}{|l|}{ Shift duty } \\
\hline Day & 0.58 & 0.40 & -0.77 & 1.92 \\
\hline Night & 0.53 & 0.54 & -1.17 & 2.23 \\
\hline Alternant & 1 & . & . & . \\
\hline \multicolumn{5}{|l|}{ Rate of non-actionable alarms } \\
\hline Less than $30 \%$ of alarms & -6.39 & $<.001$ & -8.20 & -4.58 \\
\hline Between $30 \%$ and $50 \%$ of alarms & -6.52 & $<.001$ & -8.21 & -4.82 \\
\hline Between $50 \%$ and $70 \%$ of alarms & -.060 & 0.45 & -2.17 & 0.96 \\
\hline Over $70 \%$ of alarms & 1 & . & . & . \\
\hline Responsible for alarm limit settings & & 0.01 & & \\
\hline Physician & 1.61 & & 0.35 & 2.87 \\
\hline Nurse & 1 & . & . & . \\
\hline Policies and procedures on alarm management & & 0.004 & & \\
\hline Yes & -1.86 & & -3.11 & -.61 \\
\hline No & 1 & . & . & . \\
\hline Deactivation and silencing the alarm & & $<0.001$ & & \\
\hline Yes & 4.14 & & 2.75 & 5.53 \\
\hline No & 1 & . & . & . \\
\hline Anxiety and stress & & $<0.001$ & & \\
\hline Yes & 3.14 & & 1.44 & 4.845 \\
\hline No & 1 & . & . & . \\
\hline Sleep disturbance & & 0.93 & & \\
\hline Yes & -0.08 & & -1.80 & 1.651 \\
\hline No & 1 & . & . & . \\
\hline $\begin{array}{l}\text { Bad quality of life perception } \\
\text { yes }\end{array}$ & 2.86 & 0.002 & 1.08 & 4.64 \\
\hline No & 1 & & & \\
\hline
\end{tabular}

Notes and abbreviations: 1general-purpose= general; Intensive care unit (ICU); Coronary care unit (CCU); ICU / CCU combined; Pediatric Intensive Care Unit (PICU); Neonatal Intensive Care Unit (NICU); PICU /NICU combined. 
The clinicians who declared that nurses are responsible fo alarm limit settings showed lower scores of AF than others subgroups. One of the reasons behind extreme alarms is the restriction on nurses to adjust the alarm's threshold to one that suits the patient's condition [18]. However, research proves that a nurse's main role is to manage the alarms in critical care units [25]. Cho et al. [14] reported that false alarms could be reduced if nurses actually took patients' present conditions into account and set individualized alarms ranges. The alarm load could be decreased by $40 \%$ if the nursing staff are allowed to individualize the alarm settings according to the patient [18]. Additionally, the present study revealed that ratio of patient/ staff was unsatisfactory and there is a scarcity of protocols and procedure to manage alarms- This finding is in harmony with those stated that the poor staffing and prohibitive alarm policies that prevent staff from adjusting alarm volumes or limits perpetuate the alarm fatigue [30] and maintain the trend of decreased alarm response and task performance [17]. Sliman et al. [25] reported that alarm fatigue is improving among nurses following an alarm fatigue nursing management protocol. Literature suggested that there was a lack of or limited training for alarm end users around alarm systems and alarm safety [5]. In addition, the appropriate alarm management depends on a combination of device usability, training, unit layout, IT infrastructure, and alarm management protocols and documentation capabilities [19]. So, all hospitals should develop and implement specific protocols to comply with critical care medical devices alarm [3], [18] and to minimize alarm fatigue among the clinical staff [14]. It is essential to establish a policy concerning who can choose and modify alarm limits, and clearly define what can and cannot be done under a given set of circumstances [18]. In addition, accompanying alarm fatigue management training to clinicians and other technicians covers further regulation parameters [25] .

Indeed, despite that alarm customization may increase trust in alarms and education strategies may improve response; however, they are not a panacea (solution) [30]. Other causes of false or nuisance alarms include workflows [17]- [18]; and the consumables (e.g., electrocardiogram electrodes or pulse oximeter sensors) can trigger false alarms if they are not designed for the monitor in use, if the electrodes are dry, or if they no longer adhere to the patient [18]. Sensors that are used for monitoring patient vitals may get disconnected or may malfunction [32].

\section{LIMITATIONS}

This study has several limitations. It is difficult to make a direct comparison of the scores of AF because no researcher has tried to quantify alarm fatigue in Lebanon. Most studies in $\mathrm{AF}$ evaluate the AF in critical care nurses and our sample includes nurses and physicians. There was notably an overrepresentation of nurses' staff. Nevertheless, nurses are the main professional component of the front-line staff in most health systems [33]. This overrepresentation was considered in the multivariate analysis; job position was included as an explanatory factor. It was a convenience sample. It was useful to stratify results by medical and nurses' staff. However, the sample size is relatively small and the results are limited to studied critical care units. So, the findings of this survey can be generalized with caution. It was a cross sectional study; we cannot establish a causal relationship. Finally, some psychological risks for health care workers identified by the present study (stress, anxiety, insomnia, affected nearly every aspect of daily life) are also consistent with those reported in the literature on devastating effects of COVID-19 pandemic [34].

\section{CONCLUSION}

In conclusion, inspite of longer residence time at intensive healthcare unit, physicians and nurses show similar trend reaction to alarm fatigue. Physicians score slightly higher alarm fatigue than nurses. Non-actionable alarms occurred frequently and associated to alarm fatigue. The Arabic Alarm fatigue questionnaire has a good psychometric properties and suitable to evaluate the AF in Lebanon. Multidisciplinary approaches can be established to minimize the incidence of AF. For instance, staff education, equipment (hardware and software) developments, implementation of more effective clinical protocols or guidelines, and using a valid instrument in assessing alarm fatigue all tackle the causes and effects of AF. Future implementations consist of analyzing the risk associated with the exposed hours of alarm noise, and their corresponding effects on clinicians in terms of alarm fatigue.

\section{ACKNOWLEDGMENT}

We are grateful to the subjects who participated in this survey, Mrs. F. Badran and Miss. Sally Bardan for their help and advice on the manuscript. We are grateful to the Directors of hospitals, Medical and Nursing Directors who helped to carry out this study.

\section{CONFLICTS OF INTEREST}

The authors declare no conflicts of interest regarding the publication of this paper.

\section{AUTHORS' CONTRIBUTIONS}

This work was conducted in collaboration between all the authors. AJ and $\mathrm{HB}$ managed the data collection and participated in study design and literature searches of the study. HS performed the statistical analysis and electronic form of the questionnaire, and assisted with the writing. RK, SS and ND participated in the transcultural adaption of the Alarm Fatigue questionnaire, interpretation of the data, managed literature searches. IS designed the study, managed the data analyses, and wrote the first draft of the manuscript. All authors read and approved the final manuscript. 


\section{REFERENCES}

[1] R. Joshi, H. v. d. Mortel, L. Feijs, P. Andriessen and C. v. Pul, "The heuristics of nurse responsiveness to critical patient monitor and ventilator alarms in a private room neonatal," PLOS ONE, vol. 12, no. 10, p. e0184567, 2017.

[2] M. Salous, J. Alkhawaldeh, S. Kewan, H. Aburashideh, D. B. Hani and A. Alzayyat, "Nurses' Attitudes Related To Alarm Fatigue in Critical Care Units: A Systematic Review," IOSR Journal of Nursing and Health Science, vol. 6, no. 2, pp. 62-66, 2017.

[3] S. Ashrafi, S. N. Mehri and B. Nehrir, "Designing an Alarm Fatigue Assessment Questionnaire: Evaluation of the Validity and Reliability of an Instrument," Journal of Critical Care Nursing, vol. 10, 2017.

[4] K. J. Ruskin and James P Bliss, "Alarm Fatigue and Patient Safety," TheAnesthesia Patient Safety Foundation Newsletter (APSF Newsletter). , vol. 34, no. 1, p. 1-28, 2019.

[5] T. A. Bach, L.-M. Berglund and E. Turk, "Managing alarm systems for quality and safety in the hospital setting," BMJ Open Quality, vol. 7:e000202. , 2018

[6] A. K. Sowan, A. G. Verab, E. I. Fonsecab, C. C. Reed, A. F. Tarriela and A. E. Berndt, "Nurse Competence on Physiologic Monitors Use: Toward Eliminating Alarm Fatigue in Intensive Care Units," The Open Medical Informatics Journal, vol. 11, pp. 1-11, 2017.

[7] B. J. Drew, P. Harris, J. K. Ze`gre-Hemsey, T. Mammone, D. Schindler, R. Salas-Boni, Y. Bai, A. Tinoco, Q. Ding and X. Hu., "Insights into the Problem of Alarm Fatigue with Physiologic Monitor Devices: A Comprehensive Observational Study of Consecutive Intensive Care Unit Patients," PLoS ONE 9(10): e110274., 2014.

[8] J. L. D. a. J. D. Young, "An investigation of sound levels on intensive care units with reference to the WHO guidelines," Critical Care 2013, vol. 17:R187, 2013.

[9] K. S. Simons, E. Verweij, P. M. C. Lemmens, S. Jelfs, M. Park, P. E. Spronk, J. P. C. Sonneveld, H.-M. Feijen, M. S. v. d. Steen, A. G. Kohlrausch, M. v. d. Boogaard and C. P. C. d. Jager, "Noise in the intensive care unit and its influence on sleep quality: a multicenter observational study in Dutch intensive care units," Critical Care volume 22, Article number: 250 (2018) C, vol. 22, 2018.

[10] A. C. Bridi, T. Q. Louro and R. C. L. d. Silva, "Clinical Alarms in intensive care: implications of alarm fatigue for the safety of patients," Rev. Latino-Am. Enfermagem, vol. 22, no. 6, pp. 1034-40, 2014

[11] R. J. Pugh, "The impact of noise in the intensive care unit," critical \& emergenc y care, 2007.

[12] H. Mokarami, V. Gharibi, H. O. Kalteh, M. F. Kujerdi and R. Kazemi, "Multiple environmental and psychosocial work risk factors and sleep disturbances," e.Proofing, 2020.

[13] H. Ruppel, L. D. Vaux, D. Cooper, S. Kunz, B. Duller and M. Funk, "Testing physiologic monitor alarm customization software to reduce alarm rates and improve nurses' experience of alarms in a medical intensive care unit," PLOS ONE, vol. 13, no. 10, p. 1e0205901., 2018.

[14] O. M. Cho, H. Kim, Y. W. Lee and I. Cho, "Clinical Alarms in Intensive Care Units: Perceived Obstacles of Alarm Management and Alarm Fatigue in Nurses," Healthc Inform Res. (Healthcare Informatics Research), vol. 22, no. 1, pp. 46-53, 2016

[15] S. Sendelbach and M. Funk, "Alarm Fatigue A Patient Safety Concern," AACN Advanced Critical Care, vol. 24, no. 4, pp. 378 - 386 , 2013.

[16] C. Torabizadeh, A. Yousefinya, F. Zand, M. Rakhshan and M. Fararooei, "A nurses' alarm fatigue questionnaire: development and psychometric properties," Journal of Clinical Monitoring and Computing (J Clin Monit Comput), 2016.

[17] J. Nguyen, K. Davis, G. Guglielmello and S. P. Stawicki, "Combating Alarm Fatigue: The Quest for More Accurate and Safer Clinical Monitoring Equipment," March 12th 2019.

[18] K. J. Ruskina and D. Hueske-Kraus, "Alarm fatigue: impacts on patient safety," Curr Opin Anesthesiol, vol. 28, p. 685-690, 2015.

[19] A. K. Sowan, A. F. Tarriela, T. M. Gomez, C. C. Reed and K. M. Rapp, "Nurses' Perceptions and Practices Toward Clinical Alarms in a Transplant Cardiac Intensive Care Unit: Exploring Key Issues Leading to Alarm Fatigue," JMIR Hum Factors, vol. 22, no. 1, p. e3, 2015.
[20] J. E. Bartlett, J. W. Kotrlik and C. C. Higgins, "Organizational Research: Determining Appropriate Sample Size in Survey Research," Information Technology, Learning, and Performance Journal, vol. 19, no. 1 , pp. 43-50, 2001

[21] O. o. N. i. Lebanon, "Statistics issued by the Order," Order of Nurses in Lebanon, Beirut, 2019.

[22] I. Sabbah, N. Drouby, S. Sabbah, N. Retel-Rude and M. Mercier, "Quality of life in rural and urban populations in Lebanon using SF 36 Health Survey," Health and Quality of Life Outcomes, vol. 1: 30, 2003.

[23] J. E. J. Ware, SF-36 health survey manuel and interpretation guide, Second printing ed., Boston: . , MA: The Health Institute, New England Center, 1997).

[24] H. A. Miot, "Assessing normality of data in clinical and experimental trials (editorial)," Jornal Vascular Brasileiro (J Vasc Bras. ), vol. 16, no. 2, pp. 88-91, 2017.

[25] A. M. A. F. Sliman, W. W. A. ElAziz and H. E. Mansour, "The effect of alarm fatigue nursing management protocol on critical care nurses' experience.," J Intensive Crit Care Nurs. , vol. 3, no. 4, 2020.

[26] J. L. Darbyshire and P. R. Greig, "Sleep Deprivation and Fatigue Management in the Intensive Care Unit," ICU Management \& Practice, vol. 2, pp. 137-139, 2020.

[27] I. Sabbah, H. Sabbah, S. Sabbah, H. Akoum and N. Droubi, "Burnou among Lebanese nurses: Psychometric properties of the Maslach Burnout Inventory-Human Services Survey (MBI-HSS)," Health, vol. 4, no. 9, pp. 644-652, 2012.

[28] A. Meli and S. Coppola, "The Night in the ICU," ICU Management \& Practice 2 - 2020, vol. 2, pp. 125-127, 2020.

[29] A. A. o. C. C. N. (AACN), "Managing Alarms in Acute Care Across the Life Span," $4 \quad 3$ 2018. [Online]. Available: https://www.aacn.org/clinical-resources/practice-alerts/managingalarms-in-acute-care-across-the-life-span. [Accessed 711 2020].

[30] C. Siobhán, A. Gloria and D. Maura, "Critical care nurses' knowledge of alarm fatigue and practices towards alarms: A multicentre study," Intensive and Critical Care Nursing, vol. 48, pp. 36-41, 2018.

[31] K. R. Johnson, J. I. Hagadorn and David W. Sink, "Alarm Safety and Alarm Fatigue," Clin Perinatol, vol. 44, p. 713-728, 2017.

[32] E. Özcan and D. Gommers, "Nine Nurse-Recommended Design Strategies to Improve Alarm Management in the ICU: A Qualitative Study," ICU Management \& Practice, vol. 2, pp. 129-133, 2020.

[33] I. M. Sabbah, T. T. Ibrahim, R. H. Khamis, H. A.-M. Bakhour, S. M Sabbah, N. S. Droubi and H. M. Sabbah, "The association of leadership styles and nurses well-being: a cross-sectional study in healthcare settings. 2020;36:328. [doi: ]," Pan African Medical Journal., vol. 36, no. 328, 2020.

[34] N. M. Simon, G. N. Saxe and Charles R. Marmar, "Mental Health Disorders Related to COVID-19-Related Deaths," JAMA. 2020;324(15), vol. 324, no. 15, pp. 1493-1494, 2020.

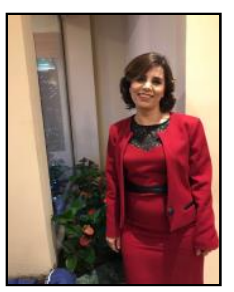

Hala Sabbah

Educational background: Ph.D. in informatics. University of Franche-Comté, Besancon, France, Research Interests:

Computer Sciences, Statistics and econometric, Multi Media, Cloud computing, Health related quality on Life.

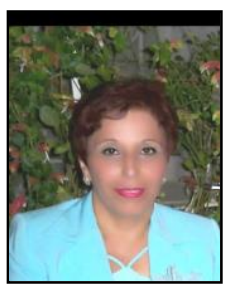

Sanaa Sabbah:

Educational background: Ph.D. in demographics. University of Franche-Comté, Besancon, France, Research Interests:

Social Sciences, demographics studies, Health related quality on Life. 
European Journal of Clinical Medicine www.ej-clinicmed.org 